\title{
Spectral and Structure Function Estimates of Turbulence Dissipation Rates in a High-Flow Tidal Channel Using Broadband ADCPs ${ }^{\mathscr{O}}$
}

\author{
Justine M. MCMillan AND Alex E. HAY \\ Department of Oceanography, Dalhousie University, Halifax, Nova Scotia, Canada
}

(Manuscript received 28 June 2016, in final form 27 September 2016)

\begin{abstract}
Spectral and structure function methods are implemented to compute the dissipation rate $\varepsilon$ from broadband, diverging-beam acoustic Doppler current profiler (ADCP) data collected at four sites in a high-flow tidal channel. This paper shows that middepth estimates of $\varepsilon$ obtained from spectral and second-order structure function (SF2) methods are both lognormally distributed with comparable means and variances. Speed binaveraged $\varepsilon$ values agree to within $16 \%$, depending on the site and tidal phase (ebb/flood). The close agreement between the two independent methods provides further support for the argument put forward by McMillan et al.: that is, that the factor-of-2 difference between shear probe and (spectral) ADCP estimates of $\varepsilon$ was likely caused by spatial differences in turbulence levels. The agreement between the spectral and both second- and third-order structure function methods also supports the use of $C_{2}=2.0$ for the SF2 universal constant. Notably, however, the SF3 method was less robust for these data. Two additional aspects of the SF2 approach are examined in some detail: 1) the differences from upstream- and downstream-facing beams are shown to arise from the Reynolds stress and 2) the inability of the ADCP to resolve small-scale motions does not affect the estimates of $\varepsilon$ but yields apparent Doppler noise levels that-counterintuitively_decrease with increasing flow speed and increasing dissipation rate. A modified SF2 method that accounts for the variance associated with the unresolved scales removes the flow speed dependence and yields noise level estimates that agree with the spectral values.
\end{abstract}

\section{Introduction}

The first statistical theories of turbulence in highReynolds-number flows were developed over 75 years ago (e.g., Taylor 1935; Kolmogorov 1941a) and were first supported by oceanographic measurements collected by Grant et al. (1962) in Discovery Passage. Additional turbulence measurements in $O\left(10^{8}\right)$ Reynolds number flows have been made only recently, stimulated in part by the emergence of in-stream tidal energy developments (Osalusi et al. 2009; Thomson et al. 2012; Richard et al. 2013; Milne et al. 2013; Hay et al. 2013; Gunawan et al. 2014; McMillan et al. 2016). To address concerns over both fatigue and extreme loads, estimates of turbulence quantities are needed at middepth (i.e., the range of depths swept out by the turbine blades, often referred to as turbine hub height). Various approaches have been implemented to overcome

Supplemental information related to this paper is available at the Journals Online website: http://dx.doi.org/10.1175/JTECH-D-16-0131.s1.

\footnotetext{
Corresponding author e-mail: Justine McMillan, justine.mcmillan@ dal.ca
}

the challenges associated with middepth turbulence measurements in high-speed (up to $6 \mathrm{~m} \mathrm{~s}^{-1}$ ) tidal currents; however, there has yet to be a standardized method for characterizing the turbulence at these high-flow sites.

In the open ocean, the dissipation rate of turbulent kinetic energy $\varepsilon$ is commonly measured in situ using shear probes; however, datasets - particularly those collected using vertical microstructure profilers - tend to be sparse and hence cannot be used to assess the intermittency in $\varepsilon$. A potential alternative is to use fast-sampling acoustic Doppler current profilers (ADCPs) to obtain remote estimates of $\varepsilon$ over longer time scales. Recent measurements reported by McMillan et al. (2016) have shown that speed bin-averaged dissipation rates computed from broadband diverging-beam ADCP data agreed to within a factor of 2 with $\varepsilon$ values obtained using shear probes mounted in the nose of an underwater streamlined buoy that was moored at middepth.

In principal, $\varepsilon$ can be estimated from diverging-beam ADCP data using either spectral or structure function

Publisher's Note: This article was revised on 20 December 2016 to correct the Collignon and Stacey reference. 
methods. Both methods require the measurement of velocity fluctuations in the inertial subrange (ISR), where the assumption of local isotropy holds. Spectral methods require a high sampling rate to ensure that the ISR is captured in time, whereas structure function (SF) methods require the flow to be isotropic over the range of spatial scales included in the spatial differences. At high Reynolds numbers, the ISR spans several wavenumber decades, implying that the assumption of local isotropy should hold over a correspondingly wide range of spatial scales. Thus, both the spectral and SF methods should be applicable in high-tidal-flow environments.

Second-order structure functions appear to have been first used to compute $\varepsilon$ from along-beam ADCP velocity measurements by Wiles et al. (2006). The method has been implemented in several subsequent turbulence investigations using Doppler profilers positioned in bottommounted configurations (Lorke 2007; Whipple and Luettich 2009; Simpson et al. 2011; Collignon and Stacey 2013; Thomson et al. 2012; Simpson et al. 2015), on mooring lines (Lucas et al. 2014; Simpson et al. 2015), and on drifting platforms (Thomson 2012). The method has been shown to give $\varepsilon$ values that are in good agreement with those obtained using acoustic Doppler velocimeters (Mohrholz et al.2008) and microstructure profilers (Wiles et al. 2006; Lucas et al. 2014). Mohrholz et al. (2008) also found the $\varepsilon$ values obtained from the third-order structure function to be consistent with the second-order estimates.

The application of SF methods specifically to broadband ADCP data warrants caution, as Doppler noise levels may contribute to high standard deviations in the instantaneous velocity differences. In addition, bin sizes of $O(1) \mathrm{m}$ may not adequately resolve the ISR and the assumption of isotropy may only be applicable over a few range bins. This isotropy assumption is particularly limiting in sheared flows, where anisotropy is argued to generate large discrepancies in the $\varepsilon$ estimates obtained along the individual beams (Wiles et al. 2006). For similar reasons, stratified conditions also limit the range over which SF methods can be applied because the production scale is limited by the Ozmidov scale. To overcome these challenges, previous studies (Mohrholz et al. 2008; Whipple and Luettich 2009; Simpson et al. 2011; Thomson 2012; Lucas et al. 2014; Simpson et al. 2015) have used pulse-coherent ADCPs, which provide high-accuracy measurements using smaller bin sizes at the expense of a reduced profiling range.

Spectral methods may—arguably—be less affected by Doppler noise than structure function methods because the mean-square sum of two positively correlated random variables is larger in amplitude than the mean-square difference. Therefore, one might expect the dissipation estimates from a spectral method to be less noisy than those from a structure function approach. Spectral methods, however, are typically applied to point measurements of two- or three-dimensional current velocities, and the application to ADCP data is limited by the orientation of the diverging ADCP beams. In standard operation, the ADCPs do not directly measure the alongstream or cross-stream velocity fluctuations, for which the theoretical spectral forms are known. Lorke and Wüest (2005) applied the inertial dissipation method to both three- and four-beam ADCP data to obtain an upper limit on $\varepsilon$ by fitting the along-beam velocity spectra in the inertial subrange to the expected form for cross-stream fluctuations. The method showed good agreement with microstructure measurements; however, a pulse-coherent mode was needed to capture the low turbulence levels $\left(\varepsilon<10^{-8} \mathrm{~W} \mathrm{~kg}^{-1}\right)$. At higher turbulence levels, dissipation rates ranging from $10^{-6}$ to $10^{-4} \mathrm{~W} \mathrm{~kg}^{-1}$ were estimated from broadband ADCP measurements by McMillan et al. (2016) using a spectral "integral method" that accounted for both Doppler noise and the orientation of the ADCP relative to the mean flow. Speed bin-averaged dissipation rates were shown to agree-to within a factor of 2-with $\varepsilon$ values obtained using shear probes moored at middepth for 2.5 days.

The purpose of this paper is to compare dissipation rates estimated using spectral and structure function methods when applied to bottom-mounted, divergentbeam, broadband ADCP data acquired at $O(10) \mathrm{m}$ ranges in a high flow, high dissipation rate tidal channel. The only other similar comparison of which we are aware is that by Lorke (2007), who compared $\varepsilon$ estimated from ADCP data via both the inertial dissipation method and the second-order SF method to results from collocated singlepoint measurements obtained with an acoustic Doppler velocimeter. In contrast to the results presented here, Lorke's data were acquired in a low-dissipation-rate $(\varepsilon<$ $10^{-8} \mathrm{~W} \mathrm{~kg}^{-1}$ ), bottom boundary layer lacustrine environment at short $O(1) \mathrm{m}$ range, using a Doppler profiler in pulse-coherent mode.

In addition to the comparisons of $\varepsilon$, we use the spectral and structure function methods to address the following questions:

1) Do estimates of $\varepsilon$ obtained using structure functions explain the factor-of-2 discrepancy between the ADCP (spectral method) and shear probe measurements obtained by McMillan et al. (2016)?

2) Can intermittency in $\varepsilon$ be assessed using broadband, diverging-beam ADCPs?

3) Do the broadband Doppler noise levels estimated from the structure function and spectral methods agree?

4) Do broadband Doppler noise levels depend on mean flow speed? 
These questions are investigated using middepth data from four different locations in a high-flow tidal channel. Relevant background information is summarized in section 2 and the dataset is described in section 3 . Results are presented in section 4 and discussed in section 5. Concluding remarks are summarized in section 6 .

\section{Theory}

\section{a. Locally isotropic turbulence}

The formal development of the statistical theory of isotropic turbulence arguably began with Taylor's (1935) paper, in which it was shown for the first time that $\varepsilon$ can be expressed in terms of the mean-square value of any component of the velocity shear. Taylor based his statistical description on the two-point autocorrelation function, for which de Kármán and Howarth (1938) derived the fundamental governing equation.

The fourth of Taylor's (1935) nine conclusions in his paper states that the Eulerian length scale of turbulence can be estimated from "simultaneous values of velocity obtained along a line" (p. 443). The structure function description - that is, velocity differences "along a line" —of isotropic turbulence was then introduced by Kolmogorov (1941a,b) in a pair of benchmark studies. In the longitudinal direction, the $n$ th-order structure function at the position $r_{0}$ is defined as

$$
D_{L^{n}}(r, t) \equiv\left\langle\left[u^{\prime}\left(r_{0}, t\right)-u^{\prime}\left(r_{0}+r, t\right)\right]^{n}\right\rangle,
$$

where $u^{\prime}$ is the fluctuating velocity component in the $r$ direction and the angled brackets represent a temporal average. Kolmogorov went on to obtain the "two-thirds law" for the second-order SF and the "four-fifths law" for the third-order SF, the latter based on the von KármánHowarth equation reexpressed in terms of structure functions (see also Pope 2000). The relationships for $D_{L L}$ and $D_{L L L}$ are given by

$$
\begin{aligned}
D_{L L} & =C_{2} \varepsilon^{2 / 3} r^{2 / 3}, \\
D_{L L L} & =-\frac{4}{5} \varepsilon r,
\end{aligned}
$$

where $C_{2}$ is a universal constant.

The spectral representation of the two-thirds law was obtained independently by Obukhov (1941) and gives the familiar $-5 / 3$ dependence of the velocity spectral density $\phi$ on the longitudinal wavenumber $k$ in the inertial subrange: that is, the range of scales for which the turbulence is locally isotropic. In one dimension, the spectra are given by

$$
\phi_{j j}=\alpha_{j} \varepsilon^{2 / 3} k^{-5 / 3}
$$

where the index $j$ corresponds to the longitudinal $(j=1)$ and transverse $(j=2,3)$ directions. The constants $\alpha_{j}$ satisfy $\alpha_{2}=\alpha_{3}=4 / 3 \alpha_{1}$.

The SF and spectral constants $C_{2}$ and $\alpha_{1}$, respectively, are related. Kolmogorov argued that the skewness of the velocity differences, given by $S^{\prime} \equiv D_{L L L} D_{L L}^{-3 / 2}$, is constant in the inertial subrange, allowing $C_{2}$ to be expressed as

$$
C_{2}=\left(-\frac{4}{5 S^{\prime}}\right)^{2 / 3}
$$

Pond et al. (1963) showed that $S^{\prime}$ can be written as

$$
S^{\prime}=-0.1 \alpha_{1}^{-3 / 2}
$$

Thus, Eq. (5) yields

$$
C_{2}=4 \alpha_{1} \text {. }
$$

The above-mentioned relationships between the spectral and structure function forms are outlined by Monin and Yaglom (1975, p. 364, 462-485), who also provide a comprehensive summary of the then-available measurements to suggest a value of $C_{2}=2.0$. More recently, Sauvageot (1992) used Doppler radar measurements of turbulence in the atmosphere to conclude that $C_{2}=2.1 \pm 0.1$. A similar range of $C_{2}=2.0 \pm 0.1$ was estimated by Saddoughi and Veeravalli (1994) using measurements obtained in a wind tunnel. Sreenivasan (1995) compiled the results from experimental studies of both grid turbulence and shear flows to conclude that the best overall agreement with Eq. (4) was obtained with $\alpha_{1}=0.5$, and therefore with $C_{2}=2.0$. Despite this finding, in recent oceanographic studies, a value of $C_{2}=2.1$ has often been adopted (e.g., Wiles et al. 2006; Lorke 2007; Simpson et al. 2011; Thomson et al. 2012; Simpson et al. 2015).

\section{b. ADCP estimates of dissipation rate}

The velocity registered along the $i$ th beam of an ADCP is the sum of a mean velocity and a fluctuating velocity $\hat{\boldsymbol{v}}_{i}^{\prime}$. The measured fluctuations are composed of both the true turbulent velocity $v_{i}^{\prime}$ and an error $e_{i}$ associated with Doppler noise (i.e., $\hat{v}_{i}^{\prime}=v_{i}^{\prime}+e_{i}$ ). It is assumed that $e_{i}$ has a Gaussian distribution with variance $\sigma_{e_{i}}^{2}$ and that the spectrum of $e_{i}$ is white with constant spectral density $N_{i}$, given by

$$
N_{i}=\frac{\sigma_{e_{i}}^{2}}{f_{N}},
$$

where $f_{N}$ is the Nyquist frequency. It is also assumed that $e_{i}$ and $v_{i}^{\prime}$ are uncorrelated. 
Spatial differences in the along-beam velocity fluctuations lead directly to longitudinal structure function estimates. If $\widehat{D}_{L L}$ is the mean-square difference in $\hat{\boldsymbol{v}}_{i}^{\prime}$, then Eqs. (1) and (2) can be combined to show that

$$
\widehat{D}_{L L}(r)=A_{i} r^{2 / 3}+B_{i},
$$

where, under the assumption of local isotropy and homogeneity of the turbulence, $A_{i}=C_{2} \varepsilon_{i}^{2 / 3}$ and $B_{i}=2 \sigma_{e_{i}}^{2}$. A least squares regression of $\widehat{D}_{L L}$ versus $r^{2 / 3}$ can be used to give

$$
\varepsilon_{i}=\left(\frac{A_{i}}{C_{2}}\right)^{3 / 2}
$$

for each of the beams. The four $\varepsilon_{i}$ estimates are averaged to give $\varepsilon_{S 2}$, that is, the estimate of dissipation rate from the second-order structure function method.

Similarly, Eqs. (1) and (3) can be combined to show that if the Doppler noise is uncorrelated with $v_{i}^{\prime}$, then the third-order moments of the along-beam velocity differences $\widehat{D}_{L L L}$ are

$$
\widehat{D}_{L L L}(r)=M_{i} r,
$$

where $M_{i}=-(4 / 5) \varepsilon_{i}$ for locally isotropic turbulence. A least squares regression of $\widehat{D}_{L L L}$ versus $r$ yields

$$
\varepsilon_{i}=-\frac{5}{4} M_{i}
$$

for each beam. Again, the four estimates of $\varepsilon_{i}$ are averaged together to give $\varepsilon_{S 3}$.

The frequency spectra $\hat{S}_{i i}(f)$ of the along-beam fluctuations $\hat{v}_{i}^{\prime}$ can be used to determine the dissipation rate using an integral method (IM) that was implemented by McMillan et al. (2016). If the ADCP is oriented such that one of the beam pairs lies in the plane of the streamwise flow, then the theoretical forms of the spectra given by Eq. (4) can be used to give

$$
\hat{S}_{T}(k)=C \varepsilon^{2 / 3} k^{-5 / 3}+N_{T},
$$

where $\hat{S}_{T}$ and $N_{T}$ are the summed wavenumber spectral densities and Doppler noise levels, respectively, which are determined using Taylor's hypothesis, that is,

$$
\begin{gathered}
\hat{S}_{T}=\frac{|U|}{2 \pi}\left(\hat{S}_{11}+\hat{S}_{22}+\hat{S}_{33}+\hat{S}_{44}\right) \\
N_{T}=\frac{|U|}{2 \pi}\left(N_{1}+N_{2}+N_{3}+N_{4}\right),
\end{gathered}
$$

where $|U|$ is the mean flow speed. The constant $C$ in Eq. (13) is given by

$$
C=\left(\frac{14}{3} \sin ^{2} \vartheta+\frac{16}{3} \cos ^{2} \vartheta\right) \alpha_{1},
$$

where $\vartheta$ is the angle of the beams with respect to the vertical. The dissipation rate can then be expressed as

$$
\varepsilon_{\mathrm{IM}}=\left[C^{-1} \overline{\left(\hat{S}_{T}-N_{T}\right) k^{5 / 3}}\right]^{3 / 2},
$$

where the overbar represents an average over the inertial subrange and the IM subscript is used to identify the method.

\section{Dataset}

Several fast-sampling ADCPs were deployed near the northern end of Grand Passage, Nova Scotia, Canada (Fig. 1). The channel is about $800 \mathrm{~m}$ wide and the flow is dominated by the $\mathrm{M}_{2}$ tide, which has a range of approximately $5 \mathrm{~m}$ and generates currents that reach $3 \mathrm{~m} \mathrm{~s}^{-1}$ near the surface. In the center of the passage, the flow is nearly rectilinear with the flood tide flowing northward and the ebb tide flowing southward. The measurements were all made during the summer, when waves tend to be small and infrequent (McMillan et al. 2016). The interaction between strong flows and several prominent bathymetric features generates intense mixing that results in a predominantly unstratified water column. CTD measurements made in July 2012 have a maximum density difference of $0.25 \mathrm{~kg} \mathrm{~m}^{-3}$ over the entire 25-m depth (Malinka 2013).

Each ADCP was deployed on a bottom pod that was ballasted using three $45-\mathrm{kg}$ lead feet. The low-profile frames were constructed from solid fiberglass rods and a fiberglass grating base. They were retrieved using an acoustic release and float line recovery system. Deployment duration varied between 3 and 12 days (Table 1). The ADCP at site 1 operated in burst mode to avoid interference with another acoustic instrument mounted on the same frame. At this site, approximately $7 \mathrm{~min}$ of data were collected every $15 \mathrm{~min}$. At the other sites, the measurements were acquired continuously.

The ADCPs were all 600-kHz RD Instruments (RDI) Workhorse instruments that recorded two-ping-averaged along-beam velocities at sampling rates between 1.49 and $1.82 \mathrm{~Hz}$ (Table 1). The ADCPs were configured to have $0.5-\mathrm{m}$ range bins with the first bin located $2.1 \mathrm{~m}$ above the bottom and the uppermost bin extending beyond the surface at high tide. For these settings (i.e., $0.5-\mathrm{m}$ range bins and two-ping ensembles), the manufacturer's instrument setup software specifies an accuracy of $\sigma_{U}=9.63 \mathrm{~cm} \mathrm{~s}^{-1}$ for the horizontal velocity; thus, the expected accuracy of the along-beam measurements is $\sigma_{v}=(\sqrt{2} \sin \vartheta) \sigma_{U}=4.66 \mathrm{~cm} \mathrm{~s}^{-1}$. 


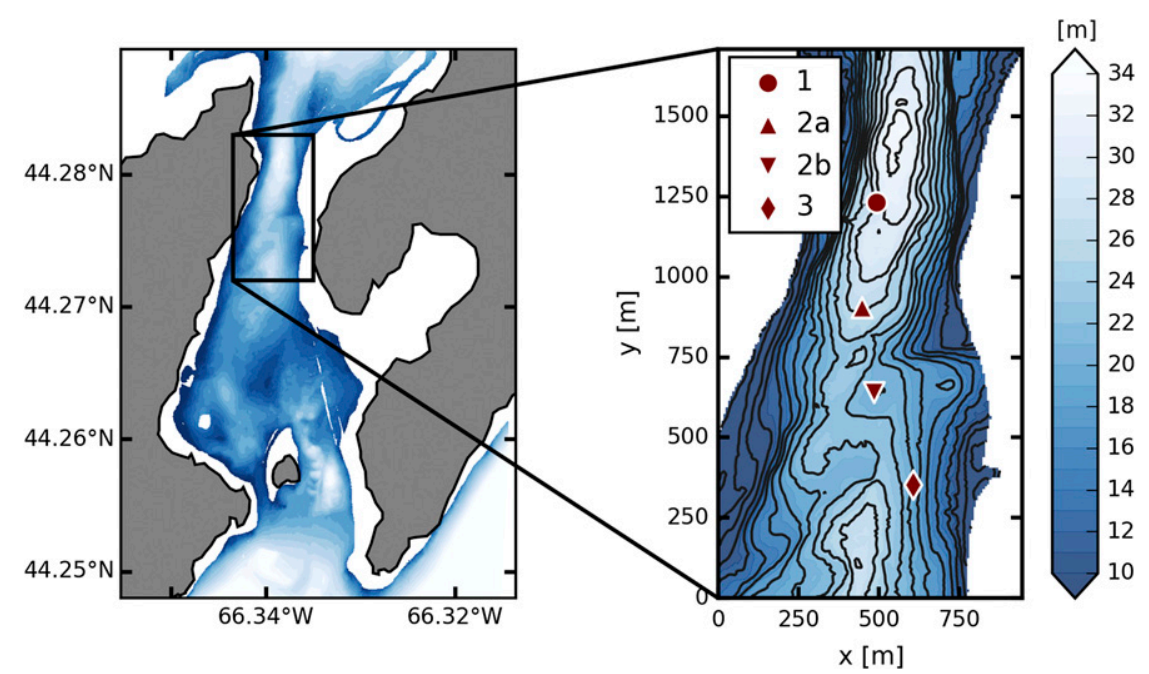

FIG. 1. Locations of fast-sampling ADCPs in Grand Passage, Nova Scotia. The contour interval at right is $2 \mathrm{~m}$.

The average attitude parameters (heading, pitch, and roll) are summarized in Table 2 . The small standard deviations in all three parameters indicate that the instrument frames remained steady throughout the chosen deployment intervals.

\section{Results}

\section{a. Mean flow}

Depending on the sampling scheme, ensemble averages of the ADCP measurements were computed for each 7-min burst (site 1) or 5-min interval (sites $2 \mathrm{a}, 2 \mathrm{~b}$, and 3). The data were then sorted into $0.2 \mathrm{~m} \mathrm{~s}^{-1}$ speed bins based on the speed $|U|$ at $z=10 \mathrm{~m}$ above bottom. Average velocity profiles for each of the deployments on the flood and ebb tides are shown in Fig. 2. All the profiles show the presence of a log layer near the bed; however, the thickness of the log layer is dependent on both the tidal phase (i.e., ebb/flood) and the instrument location. On the flood tide, the velocity profiles are logarithmic beyond $z=15 \mathrm{~m}$ at sites $1,2 \mathrm{a}$, and $2 \mathrm{~b}$, whereas the $\log$ layer terminates at $z \approx 5 \mathrm{~m}$ at site 3 . The opposite trend is observed on the ebb tide, that is, the log layer is thickest at site 3 . This asymmetry is attributed primarily to the presence of a ridge on the eastern side of the passage between sites $2 \mathrm{a}$ and $2 \mathrm{~b}$ (Fig. 1). As the flow passes over the ridge, macroturbulence is generated and advected downstream, thickening the boundary layer (Hay et al. 2013; McMillan et al. 2016).

For the purposes of this paper, the analysis will focus on the measurements at middepth, which was chosen to be $10 \mathrm{~m}$ above bottom at all sites. A vertical averaging interval of $4.5 \mathrm{~m}$ (i.e., nine range bins) was used (Fig. 2, gray region), and the depth-averaged horizontal velocity over this interval is denoted by $U_{r}$. This depth interval was chosen because along-beam velocity spectra (e.g., shown in McMillan et al. 2016) for $\left|U_{r}\right|>1 \mathrm{~m} \mathrm{~s}^{-1}$ exhibit a $k^{-5 / 3}$ range for scales below $5 \mathrm{~m}$; thus, the assumption of local isotropy, which is fundamental for both the spectral and structure function methods, is applicable over this depth interval.

The principal flood and ebb directions of $U_{r}$ were computed using empirical orthogonal functions and are shown relative to both true north and the ADCP beam axes in Fig. 3. With the exception of site 2a, the frames

TABLE 1. Summary of the fast-sampling RDI 600-kHz ADCP deployments in Grand Passage.

\begin{tabular}{|c|c|c|c|c|c|c|c|}
\hline Site & Start date & Lon $\left(^{\circ}\right)$ & Lat $\left(^{\circ}\right)$ & Depth (m) & Duration (days) & Sample rate $(\mathrm{Hz})$ & Sampling method \\
\hline 1 & 30 Jul 2013 & -66.3391 & 44.2799 & 28 & $7^{\mathrm{a}}$ & 1.49 & Burst \\
\hline $2 \mathrm{a}$ & 27 Jun $2013^{b}$ & -66.3395 & 44.2778 & 29 & 11.5 & 1.67 & Continuous \\
\hline $2 b$ & 27 Jun $2013^{b}$ & -66.3391 & 44.2761 & 23 & 11.8 & 1.67 & Continuous \\
\hline 3 & 4 Sep 2012 & -66.3380 & 44.2743 & 22 & 3 & 1.82 & Continuous \\
\hline
\end{tabular}

\footnotetext{
${ }^{\mathrm{a}}$ The ADCP was deployed for 9 days, but the last 2 days were omitted from analysis due to low correlations.

${ }^{\mathrm{b}}$ The ADCPs were deployed 7 days earlier but shifted after 1 week. The most stable interval was chosen for analysis.
} 
TABLE 2. Summary of the attitude parameters for the ADCP deployments.

\begin{tabular}{llrr}
\hline \hline Site & Heading $\left(^{\circ}\right)$ & \multicolumn{1}{c}{ Pitch $\left({ }^{\circ}\right)$} & \multicolumn{1}{c}{ Roll $\left(^{\circ}\right)$} \\
\hline 1 & $202.5 \pm 0.4$ & $3.87 \pm 0.05$ & $-4.40 \pm 0.10$ \\
2a & $342.8 \pm 0.3$ & $-3.31 \pm 0.10$ & $-7.02 \pm 0.05$ \\
2b & $196.0 \pm 0.2$ & $3.80 \pm 0.02$ & $2.55 \pm 0.01$ \\
3 & $289.5 \pm 0.3$ & $-7.88 \pm 0.03$ & $0.30 \pm 0.03$ \\
\hline
\end{tabular}

were well aligned with the streamwise flow direction. The deviations between the mean flow direction and the axis of the closest beam pair at sites $3,2 b$, and 1 were $0.04^{\circ}, 1.3^{\circ}$, and $15^{\circ}$, respectively.

\section{b. Dissipation rates from the second-order SF method}

For each ensemble (7 min at site 1, 5-min otherwise), along-beam differences in the velocity fluctuations were computed about $z=10 \mathrm{~m}$ to obtain estimates of $\widehat{D}_{L L}$. Because the velocities measured in adjacent bins are not independent, differences were computed for all possible combinations of along-beam distance $r$ ranging from $1.1 \mathrm{~m}$ (two bins) to $4.3 \mathrm{~m}$ (nine bins) within the averaging region. This method resulted in 28 values of $\widehat{D}_{L L}$ and differs from the center-differencing scheme described by Wiles et al. (2005) and subsequently implemented in other studies (e.g., Wiles et al. 2006; Mohrholz et al. 2008; Lanckriet and Puleo 2013). It was confirmed that the center-differencing scheme, which would have yielded only 10 values of $\widehat{D}_{L L}$, gives comparable estimates of the dissipation rate. However, because the approach adopted here makes use of all possible values of $\widehat{D}_{L L}$ at a given separation, it results in tighter confidence intervals for the estimates of $\varepsilon_{i}$ and $\sigma_{e_{i}}$. Using all possible values at a given separation is also consistent with the assumption that the turbulence properties are homogeneous and isotropic.

A least squares regression to Eq. (9) was performed for each ensemble, and the $\varepsilon_{i}$ value for each beam was obtained using Eq. (10) with $C_{2}=2.0$ (see supplementary material for an example). The averages of the four $\varepsilon_{i}$ values are plotted as purple points in Fig. 4. The uncertainty, $\Delta \varepsilon_{i}$, was estimated from the $95 \%$ confidence intervals on the slope and data were rejected if $\Delta \varepsilon_{i} / \varepsilon_{i}>0.6$. Values were also rejected if $B_{i}<0$, which corresponds to a negative estimate of $\sigma_{e_{i}}$. When taking the average of $\varepsilon_{i}$ computed from all four beams, the data rejection rate for $\left|U_{r}\right|>0.8 \mathrm{~m} \mathrm{~s}^{-1}$ ranged from $4 \%$ to $34 \%$ (Table 4 ).

\section{c. Dissipation rates from the spectral method}

For each ensemble ( $7 \mathrm{~min}$ at site $1,5 \mathrm{~min}$ otherwise), $\hat{S}_{i i}(f)$ were computed for each vertical bin using Hanningwindowed intervals of 20 points (approximately 11-13 s) with $50 \%$ overlap. This resulted in spectra with a frequency resolution of approximately $0.075 \mathrm{~Hz}$ and up to 100 degrees of freedom (Nuttall 1971). Spectra were then averaged over the $4.5-\mathrm{m}$ range interval centered at $z=10 \mathrm{~m}$.
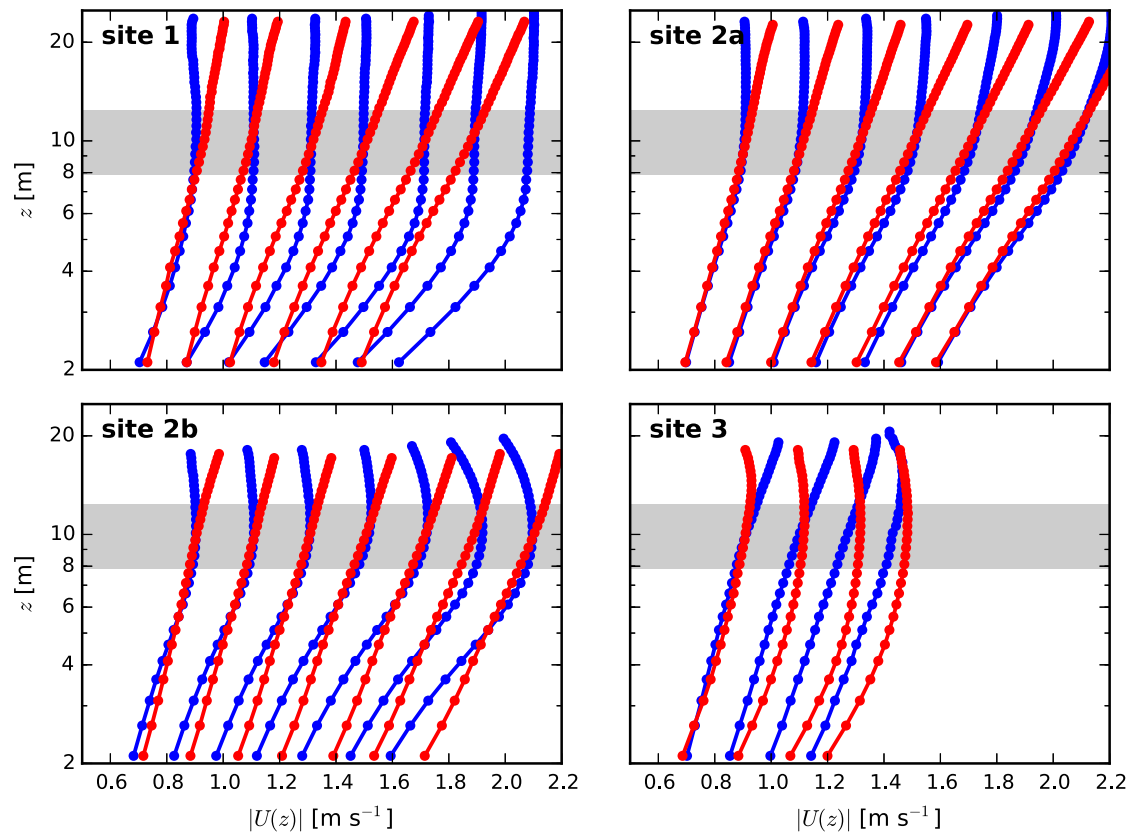

FIG. 2. Speed bin-averaged velocity profiles during flood (red) and ebb (blue) at each site. The gray region identifies the depth range over which the dissipation rates were calculated. 

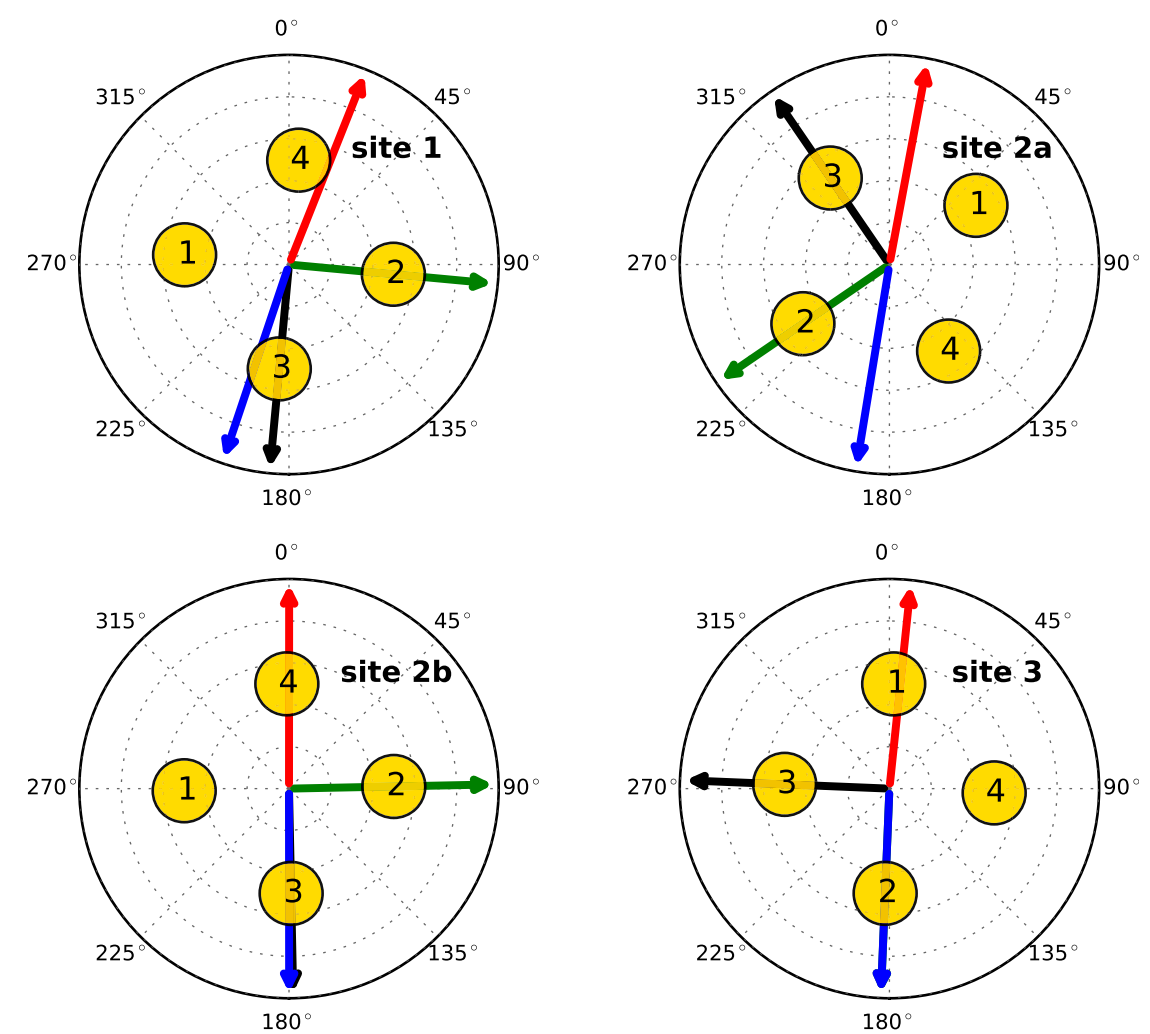

FIG. 3. Instrument orientation relative to true north $\left(0^{\circ}\right)$. Principal flow directions of the mean velocity at the 10-m reference height $\left(U_{r}\right)$ are shown in red and blue for the flood and ebb tides, respectively. The transducer faces are indicated by the yellow circles and numbered according to the RDI convention. The instrument's $X$ and $Y$ axes are shown in black $\left(v_{X}\right)$ and green $\left(v_{Y}\right)$, respectively.

For each beam $N_{i}$ was estimated by computing the average spectral density from $f \in\left[0.5 f_{N}, f_{N}\right]$ for the lowest flow speeds $\left(\left|U_{r}\right|<0.25 \mathrm{~m} \mathrm{~s}^{-1}\right)$. The corresponding uncertainties $\sigma_{e_{i}}$ were computed using Eq. (8), and the four-beam mean $\bar{\sigma}_{e_{i}}$ and standard deviation $\Delta \sigma_{e_{i}}$ at each site are summarized in Table 3 . These values are all within $6 \%$ of the expected accuracy of $\sigma_{v}=4.66 \mathrm{~cm} \mathrm{~s}^{-1}$.

The dissipation rate $\varepsilon_{\mathrm{IM}}$ was computed by applying Eq. (17) over the inertial subrange given by $k \in$ $[1.1,2.1] \mathrm{rad} \mathrm{m}^{-1}$ (see supplementary material for an example). The same wavenumber interval was used for all ensembles. Time series of the $\varepsilon_{\mathrm{IM}}$ values exhibit a clear tidal modulation at all sites (Fig. 4, green points).

At low flow speeds, the low-frequency resolution of the spectra resulted in only one spectral value in the inertial subrange. Consequently, data for these flow speeds were not given further consideration. Data were also rejected for ensembles for which the spectral shape of $\hat{S}_{T}-N_{T}$ deviated from the expected $-5 / 3$ slope in the inertial subrange by more than $20 \%$, that is,

$$
\frac{|\gamma|}{5 / 3}>0.2
$$

where $\gamma$ is defined such that $\left(\hat{S}_{T}-N_{T}\right) \sim k^{-5 / 3+\gamma}$. For all sites, nearly half of the data for $\left|U_{r}\right|>0.8 \mathrm{~m} \mathrm{~s}^{-1}$ were rejected on the basis of spectral shape (Table 4).

\section{d. Comparison of IM and SF2 dissipation rates}

The time series of the $\varepsilon$ values (Fig. 4) from the second-order structure function method (purple points) and the spectral integral method (green points) track each other closely. These estimates are plotted one against the other in Fig. 5 (gray points). The points corresponding to individual estimates are scattered about the 1:1 line and are typically within a factor of 2 of each other (dashed lines). Speed bin-averaged dissipation rates were determined by averaging the $\varepsilon$ values within $0.2 \mathrm{~m} \mathrm{~s}^{-1}$ speed bins (Fig. 5, red and blue markers). The least squares best-fit lines indicate that the $\varepsilon_{S 2}$ values tend to be within $16 \%$ of the $\varepsilon_{\mathrm{IM}}$ values, with the tendency that $\varepsilon_{S 2}<\varepsilon_{\mathrm{IM}}$. The agreement between $\varepsilon_{\mathrm{IM}}$ and $\varepsilon_{S 2}$ at all four sites suggests that the methods are insensitive 


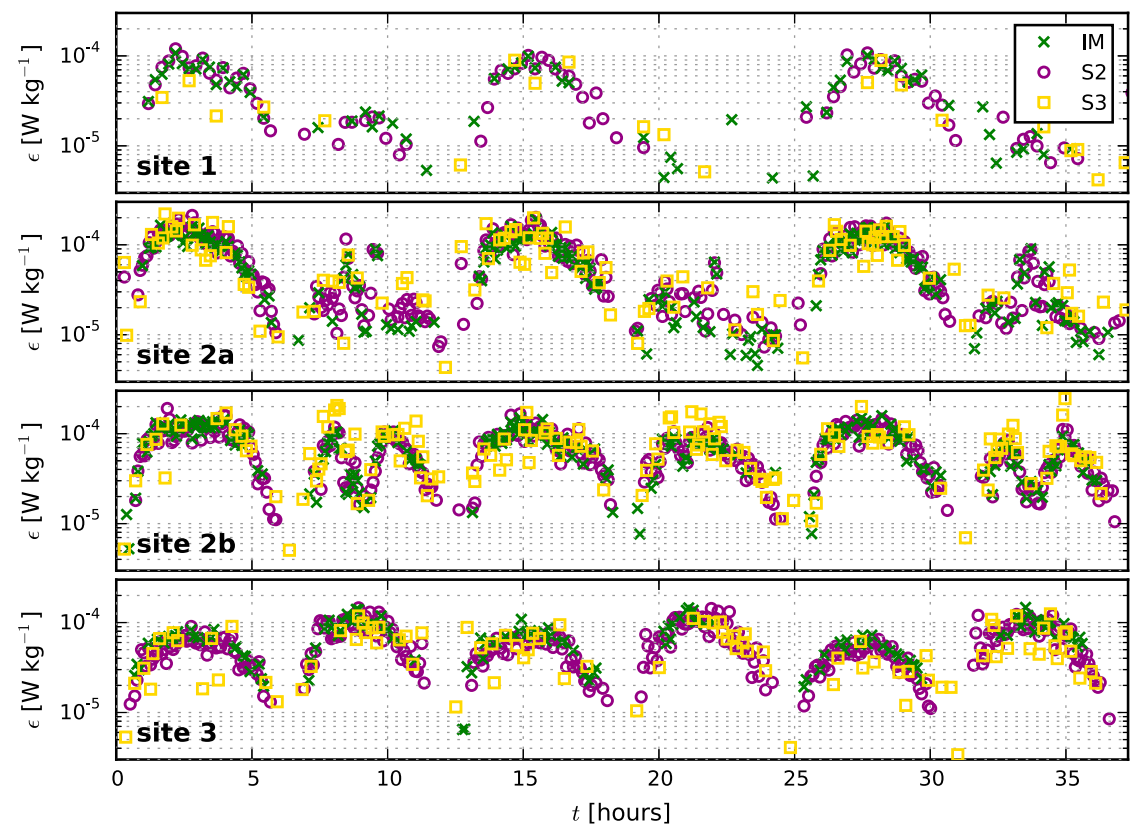

FIG. 4. The computed dissipation rates over three tidal cycles at each location. In all panels, $t=0$ coincides with the start of a flood tide. Gaps in the data correspond to ensembles for which the data were rejected (see Table 4).

to the orientation of the ADCP. This is somewhat surprising, as the IM was derived under the assumption that one of the beam pairs is oriented close to the streamwise direction (McMillan et al. 2016).

\section{e. Dissipation rate PDFs}

The probability density functions (PDFs) of $x=$ $\log _{10} \varepsilon$-computed for $\left|U_{r}\right|>0.75 \max \left(\left|U_{r}\right|\right)$-are plotted in Fig. 6 . The values for the mean $\bar{x}$ and standard deviation $\sigma_{x}$ of the PDFs are very similar for the IM and SF2 methods at each of the sites (Table 5). In a similar comparison between the inertial dissipation method and the SF2 method, Lorke (2007) obtained lognormal distributions of $\varepsilon$ using 21-min temporal averages of pulsecoherent Doppler profiler data. The distributions for

TABLE 3. Summary of the computed Doppler noise levels. The IM column gives the four-beam mean $\bar{\sigma}_{e_{i}}$ and standard deviation $\Delta \sigma_{e_{i}}$ as computed from the velocity spectra at low flow speeds. The SF2 column gives the corrected values determined via Eq. (26), where the statistics are for all beams and all speed bins. The manufacturer-specified accuracy is $4.66 \mathrm{~cm} \mathrm{~s}^{-1}$.

\begin{tabular}{lcc}
\hline \hline & $\mathrm{IM}$ & $\frac{\mathrm{SF} 2}{}$ \\
\cline { 2 - 3 } Site & $\begin{array}{c}\bar{\sigma}_{e_{i}} \pm \Delta \sigma_{e_{i}} \\
\left(\mathrm{~cm} \mathrm{~s}^{-1}\right)\end{array}$ & $\begin{array}{c}\overline{\tilde{\sigma}}_{e_{i}} \pm \Delta \tilde{\sigma}_{e_{i}} \\
\left(\mathrm{~cm} \mathrm{~s}^{-1}\right)\end{array}$ \\
\hline 1 & $4.58 \pm 0.08$ & $4.55 \pm 0.12$ \\
$2 \mathrm{a}$ & $4.66 \pm 0.04$ & $4.64 \pm 0.11$ \\
$2 \mathrm{~b}$ & $4.81 \pm 0.03$ & $4.77 \pm 0.09$ \\
3 & $4.92 \pm 0.02$ & $4.98 \pm 0.08$ \\
\hline
\end{tabular}

both methods had the same variance; however, the mean value was 2.5 times greater for the SF2 method. For the present results, on the other hand, the mean values from the two methods are in close agreement (Table 5), differing in linear space by at most $23 \%$ in one instance (site 3 , flood) and by less than $5 \%$ in the remaining seven instances, with no systematic bias.

The variance $\sigma_{x}^{2}$ of the PDFs is dependent on both the site and the tidal phase (i.e., ebb/flood). The widest distributions occur on the ebb tide at sites 1, 2a, and $2 \mathrm{~b}$. At these locations the vertical averaging region is just above the logarithmic layer on the ebb tide (Fig. 2), suggesting that time variations in the boundary layer thickness lead to increased variability in $\varepsilon$. The intermittency exponent $\mu$ is also larger because $\sigma_{x}^{2}=\ln A+\mu \ln (\mathscr{L} / r)$, where $A$ is a positive constant (Pope 2000).

Standardized PDFs are shown in Fig. 7 for two speed ranges and compared to the lognormal distribution that is expected for a high-Reynolds-number flow. For each

TABLE 4. Percentage of data rejected for flows with $\left|U_{r}\right|>0.8 \mathrm{~m} \mathrm{~s}^{-1}$.

\begin{tabular}{lccc}
\hline \hline Site & IM & SF2 & SF3 \\
\hline 1 & 44 & 34 & 84 \\
$2 \mathrm{a}$ & 42 & 21 & 77 \\
$2 \mathrm{a}$ & 40 & 10 & 72 \\
3 & 58 & 4 & 76 \\
\hline
\end{tabular}



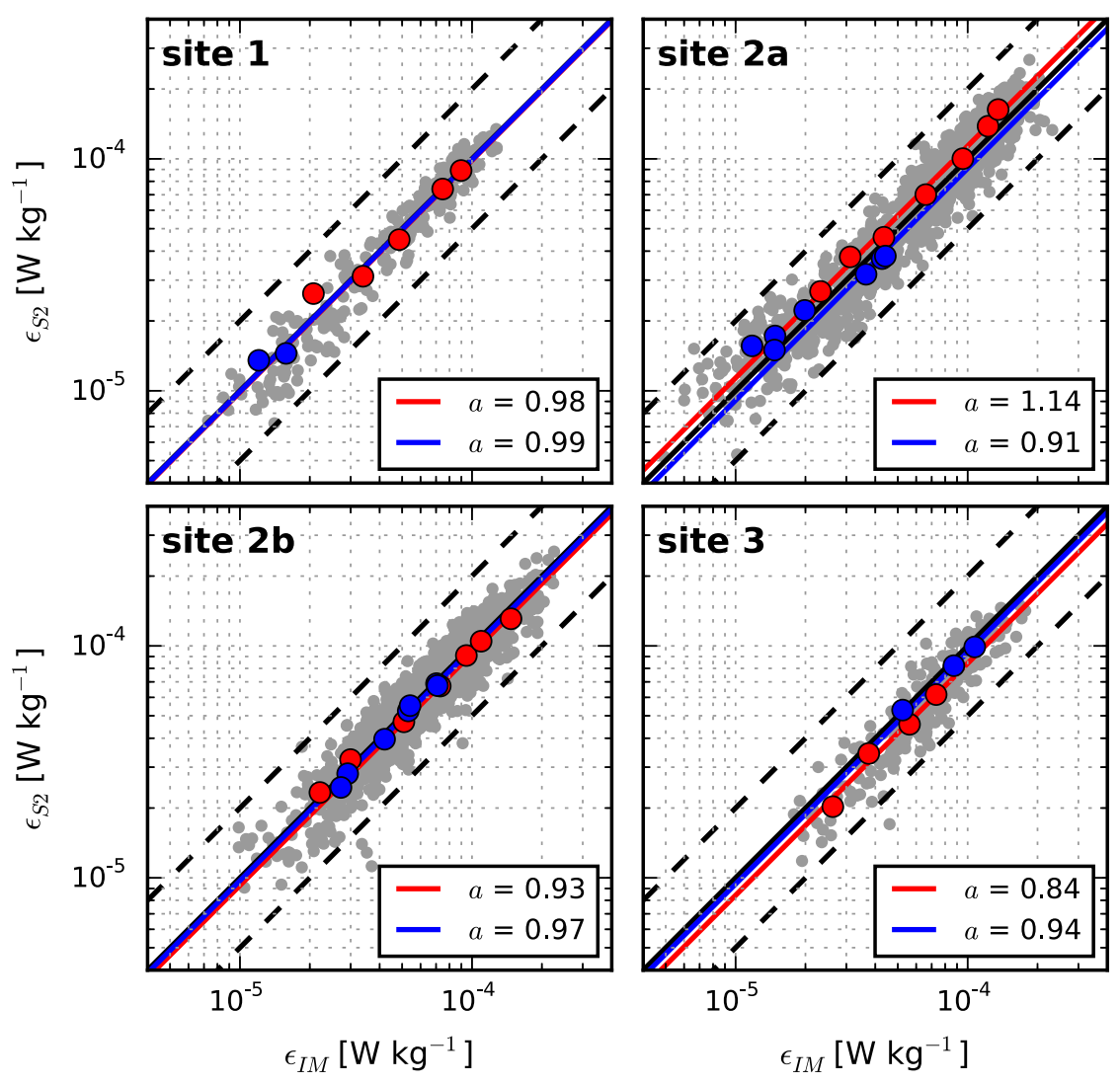

FIG. 5. Comparison of the dissipation rates at each site as computed from the second-order structure function $\left(\varepsilon_{S 2}\right)$ and the integral method $\left(\varepsilon_{\mathrm{IM}}\right)$. Averages within $0.2 \mathrm{~m} \mathrm{~s}^{-1}$ speed bins are plotted in red and blue for the flood and ebb tides, respectively. The values of the best-fit slope, $a$, to the speed bin averages are shown in the legend. The 1:1 line is solid black, whereas the 1:2 and 2:1 lines are dashed.

site, tidal phase, and speed range, values of $y=$ $\sigma_{x}^{-1}(x-\bar{x})$ were computed. The values for ebb and flood at all four sites were then combined to yield better statistics. The resulting PDFs (Fig. 7) for both the IM and SF2 methods agree well with the lognormal distributions for both speed ranges. This further indicates that the intermittency in the dissipation rate is remotely detectable in high-speed flows using diverging-beam ADCPs.

\section{f. Dissipation rates from the third-order SF method}

Third-order structure functions, $\widehat{D}_{L L L}$, were computed using the same method and depth range described in section $4 \mathrm{~b}$. The values were regressed against Eq. (11) to determine $\varepsilon_{i}$ using Eq. (12). The four $\varepsilon_{i}$ values were then averaged to give $\varepsilon_{S 3}$ and are plotted as yellow squares in Fig. 4, which shows that the values track the $\varepsilon_{S 2}$ estimates reasonably well. Data were rejected if $M_{i}<0$, which gives a negative value for $\varepsilon_{i}$. This criterion resulted in $72 \%-84 \%$ of the $\varepsilon_{S 3}$ estimates being rejected, indicating that for these data, the third-order method is much less robust, despite the form of $\widehat{D}_{L L L}$, given by Eq. (11), being independent of $\sigma_{e_{i}}$.

Despite the high percentage of data rejected for the third-order method, a comparison of $\varepsilon_{S 3}$ and $\varepsilon_{S 2}$ is relevant. The estimates from all four sites are plotted one against the other in Fig. 8. The points are scattered about the 1:1 line, with the tendency that $\varepsilon_{S 3}>\varepsilon_{S 2}$. This bias was exhibited at all four sites. Because the theoretical form of the third-order structure function, given by Eq. (3), is known exactly and $\widehat{D}_{L L L}$ is independent of noise, these results support the use of a value of $C_{2}=2.0$. The $\varepsilon_{S 2}$ estimates would be $7 \%$ lower, resulting in a larger discrepancy between $\varepsilon_{S 3}$ and $\varepsilon_{S 2}$, if a value of $C_{2}=2.1$ had been used instead.

\section{Discussion}

\section{a. The discrepancy with shear probe estimates}

One of the objectives of work related to this paper was to determine whether the factor-of-2 discrepancy 


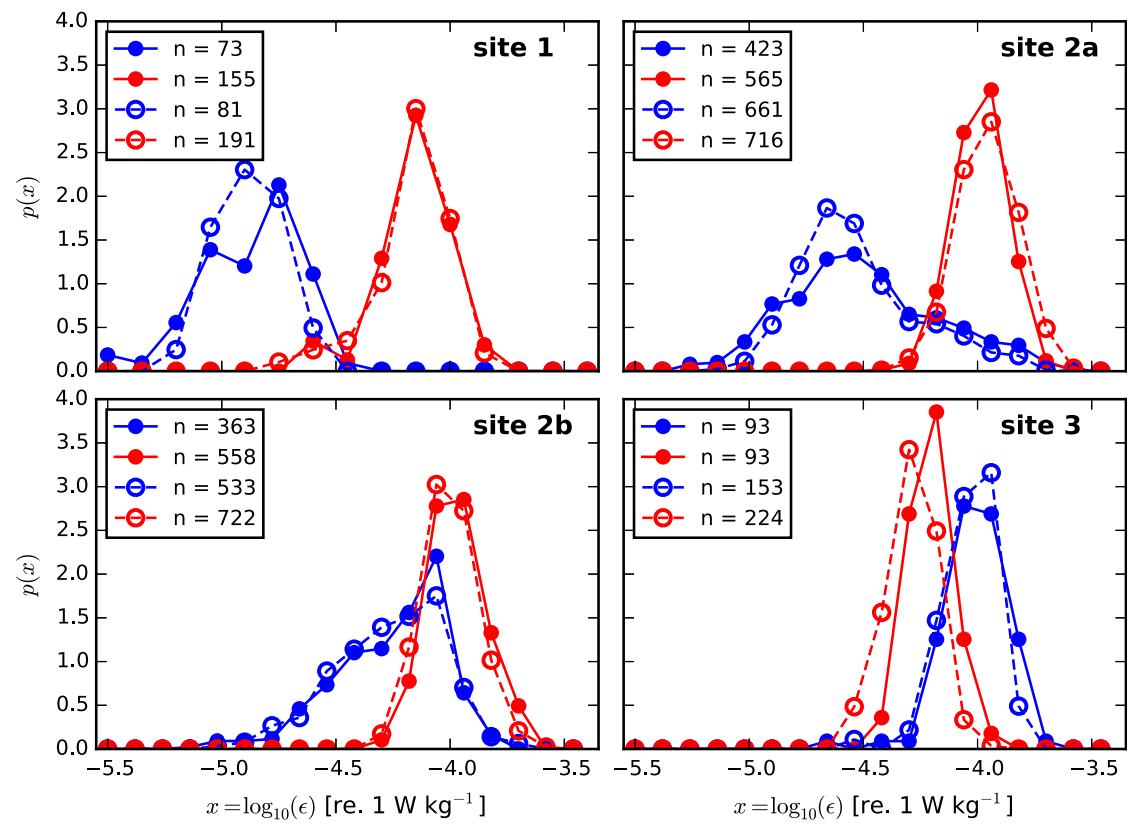

FIG. 6. Histograms of dissipation rates for $\left|U_{r}\right|>0.75 \max \left(\left|U_{r}\right|\right)$. Solid lines and filled circles indicate the IM, and the dashed lines and open circles indicate the SF2 method. Blue and red indicate ebb and flood tides, respectively. The number of ensembles $n$ for each PDF is indicated in the legend. The $\max \left(\left|U_{r}\right|\right)$ values at sites $1,2 \mathrm{a}, 2 \mathrm{~b}$, and 3 are 2.1, 2.1, 2.2, and $1.5 \mathrm{~m} \mathrm{~s}^{-1}$, respectively.

between the shear probe and ADCP estimates of $\varepsilon$ reported in McMillan et al. (2016) could be attributed to the use of a spectrum-based method. The agreement between the IM and SF2 methods in both the means (Fig. 5) and the PDFs (Fig. 6) indicates otherwise. In addition, the third-order estimates, which are unaffected by noise and do not involve a universal constant, were higher than $\varepsilon_{S 2}$ (Fig. 8) and thus in greater disagreement with the shear probe results. These conclusions are further evidence that the observed differences are real and are likely explained by the 40-m spatial separation of the instruments, as McMillan et al. (2016) suggested.

The mean $\bar{x}$ and standard deviation $\sigma_{x}$ of the shear probe estimates of $x=\log _{10} \varepsilon$ are included in Table 5 for additional comparison. As expected, based on McMillan et al. (2016), the statistics are more different between the shear probe (SP) site and nearby site 1 than between the SF2 method and the IM applied to the site 1 data. However, the result that a higher mean and lower variance were observed on the flood tide compared to the ebb is consistent between both the shear probe and ADCP estimates of $\varepsilon$. Spatial variability in $\varepsilon$ within the passage is also apparent in the differences between ADCP sites $2 a$ and $2 b$. The ADCPs at these sites were deployed concurrently about $100 \mathrm{~m}$ apart in the streamwise direction (Fig. 1). On the flood tide, both $\bar{x}$ and $\sigma_{x}$ are comparable, whereas on the ebb tide the dissipation rates are lower but more variable at site $2 \mathrm{a}$.

\section{b. Streamwise biases in $\varepsilon_{S 2}$ estimates}

Wiles et al. (2006) found that the dissipation rates were consistently higher for the upstream-facing beam. Dissipation estimates from the upstream- and downstreamfacing beams in the present dataset are compared in Fig. 9. Speed bin-averaged $\varepsilon_{i}$ estimates from the upstream-facing beam tend to be greater than the downstream-facing beam by a factor ranging from 1.27 to 1.71 , consistent with the observation by Wiles et al. (2006). Thus, there is a tendency for dissipation rates estimated via the SF2 method to be biased high in the upstream-facing direction relative

TABLE 5. Mean $\bar{x}$ and standard deviations $\sigma_{x}$ of $x=\log _{10} \varepsilon$. Values are given with reference to $1 \mathrm{~W} \mathrm{~kg}^{-1}$. The shear probe (SP) site was located more than $40 \mathrm{~m}$ to the east of site 1 and was the location of the shear probe measurements reported in McMillan et al. (2016).

\begin{tabular}{|c|c|c|c|c|c|}
\hline \multirow[b]{2}{*}{ Site } & \multirow[b]{2}{*}{$\varepsilon$} & \multicolumn{2}{|c|}{ Flood } & \multicolumn{2}{|c|}{ Ebb } \\
\hline & & $\bar{x}$ & $\sigma_{x}$ & $\bar{x}$ & $\sigma_{x}$ \\
\hline SP & - & -4.28 & 0.12 & -5.11 & 0.26 \\
\hline \multirow[t]{2}{*}{1} & $\varepsilon_{\mathrm{IM}}$ & -4.16 & 0.15 & -4.88 & 0.22 \\
\hline & $\varepsilon_{S 2}$ & -4.17 & 0.17 & -4.87 & 0.14 \\
\hline \multirow[t]{2}{*}{$2 \mathrm{a}$} & $\varepsilon_{\mathrm{IM}}$ & -3.99 & 0.11 & -4.50 & 0.33 \\
\hline & $\varepsilon_{S 2}$ & -3.96 & 0.13 & -4.52 & 0.26 \\
\hline \multirow[t]{2}{*}{$2 b$} & $\varepsilon_{\mathrm{IM}}$ & -3.98 & 0.12 & -4.26 & 0.24 \\
\hline & $\varepsilon_{S 2}$ & -4.00 & 0.12 & -4.27 & 0.24 \\
\hline \multirow[t]{2}{*}{3} & $\varepsilon_{\mathrm{IM}}$ & -4.20 & 0.09 & -4.01 & 0.14 \\
\hline & $\varepsilon_{S 2}$ & -4.29 & 0.11 & -4.03 & 0.12 \\
\hline
\end{tabular}



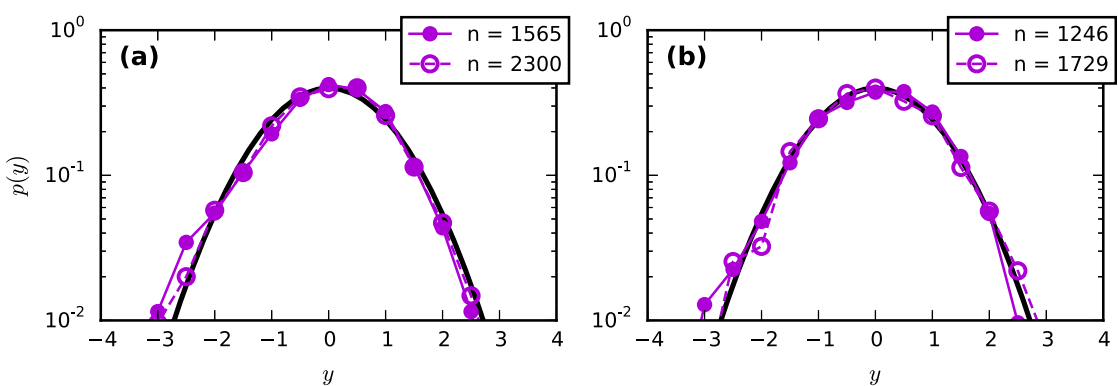

FIG. 7. Standardized PDFs of $y=\sigma_{x}^{-1}(x-\bar{x})$ for (a) $1.4<\left|U_{r}\right|<1.8 \mathrm{~m} \mathrm{~s}^{-1}$ and (b) $1.8<\left|U_{r}\right|<2.2 \mathrm{~m} \mathrm{~s}^{-1}$, where $x=\log _{10} \varepsilon$. Solid lines and filled circles indicate the IM, and dashed lines and open circles indicate the SF2 method. The number of ensembles $n$ for each PDF is indicated in the legend, and the black curves are the normal distributions.

to the downstream-facing direction. An explanation for this result is needed and is provided below.

Consider an ADCP oriented such that beam 1 faces upstream into the flow and beam 2 downstream, then from Eq. (1) $D_{L L}$ for each beam would be given by

$$
\begin{aligned}
& D_{1}=D_{u} \sin ^{2} \vartheta+D_{w} \cos ^{2} \vartheta-2 D_{u w} \sin \vartheta \cos \vartheta \\
& D_{2}=D_{u} \sin ^{2} \vartheta+D_{w} \cos ^{2} \vartheta+2 D_{u w} \sin \vartheta \cos \vartheta
\end{aligned}
$$

where $D_{u}$ and $D_{w}$ are the second-order moments of the streamwise and vertical velocity differences, respectively, and $D_{u w}$ is given by

$$
D_{u w}=\overline{u_{0}^{\prime} w_{0}^{\prime}}+\overline{u_{r}^{\prime} w_{r}^{\prime}}-\overline{u_{r}^{\prime} w_{0}^{\prime}}-\overline{u_{0}^{\prime} w_{r}^{\prime}}
$$

where $u^{\prime}$ and $w^{\prime}$ are the turbulent velocities at the positions denoted by the subscripts [e.g., $\left.u_{0}^{\prime}=u^{\prime}\left(r_{0}\right)\right]$. Because the correlations at spatially separated points are expected to be less than the correlations at the same point, $D_{u w}$ can be approximated as

$$
D_{u w} \approx \overline{u_{0}^{\prime} w_{0}^{\prime}}+\overline{u_{r}^{\prime} w_{r}^{\prime}} \approx 2 \overline{u^{\prime} w^{\prime}}
$$

Invoking the standard assumption of horizontal homogeneity of second-order moments of the turbulence (e.g., Stacey et al. 1999), Eqs. (19), (20), and (22) can be combined to give $D_{1}-D_{2} \sim-4 \overline{u^{\prime} w^{\prime}}$. Since $\overline{u^{\prime} w^{\prime}}<0$ within the log layer, the upstream-facing beam is expected to yield a higher dissipation rate than the downstream-facing beam. This result is consistent with the lines of best fit shown in Fig. 9: high Reynolds stresses of $-\overline{u^{\prime} w^{\prime}} \sim 3 \times 10^{-3} \mathrm{~m}^{2} \mathrm{~s}^{-2}$ are associated with the flood tides at sites 1 and $2 b$, and with the ebb tide at site 3 . These high Reynolds stresses, which yield equivalent dissipation rates of approximately $3 \times 10^{-5} \mathrm{~W} \mathrm{~kg}^{-1}$ via Eq. (2), occur when the middepth location $(z=10 \mathrm{~m})$ is within the logarithmic layer (Fig. 2).
Assuming statistical homogeneity across the beams, the average of Eqs. (19) and (20) can be computed to eliminate the $D_{u w}$ terms. This demonstrates that averaging the along-beam estimates of $\varepsilon_{i}$ is important for achieving accurate results when implementing the structure function methods, for diverging-beam ADCPs in situations where the Reynolds stress is significant.

\section{c. Doppler noise levels}

In the implementation of the IM, the standard deviation of the Doppler noise $\sigma_{e_{i}}$ is determined for each beam from the spectral levels at high frequencies and low flow speeds (Table 3). To estimate $\varepsilon_{\text {IM }}$ via Eq. (17),

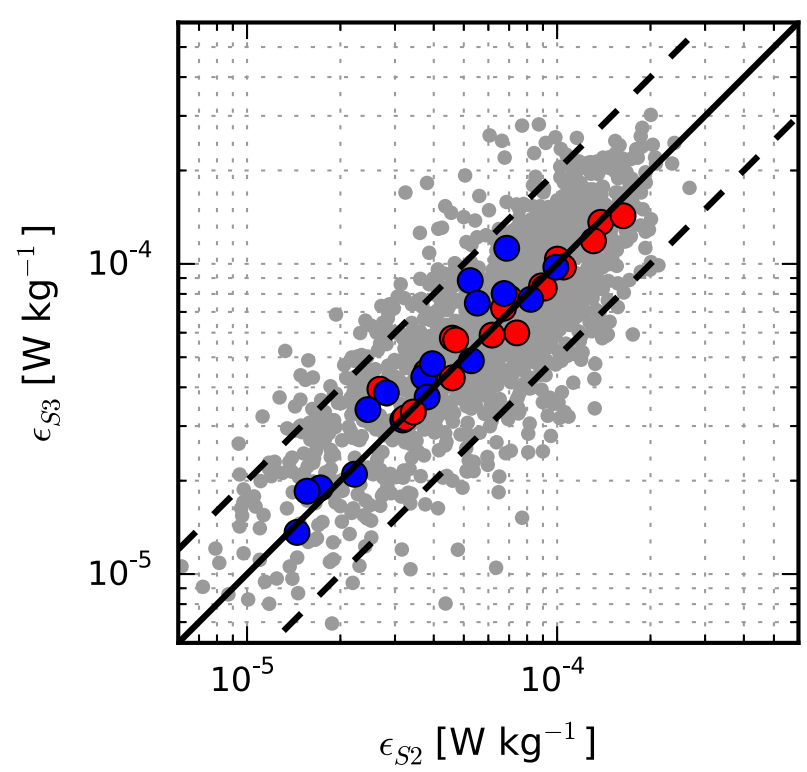

FIG. 8. Comparison of the dissipation rates at all sites as computed from the third-order $\left(\varepsilon_{S 3}\right)$ and second-order $\left(\varepsilon_{S 2}\right)$ structure functions. Averages within $0.2 \mathrm{~m} \mathrm{~s}^{-1}$ speed bins were computed separately for each site and are plotted in red and blue for the flood and ebb tides, respectively. The 1:1 line is solid black, whereas the 1:2 and 2:1 lines are dashed. 

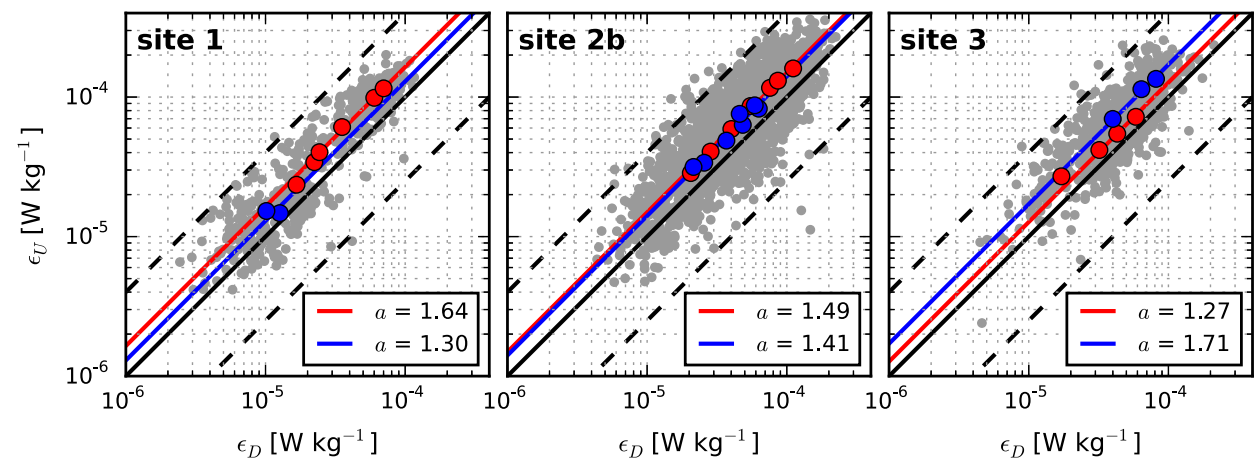

FIG. 9. Comparisons of $\varepsilon_{i}$ determined using the SF2 method for the upstream-facing $\left(\varepsilon_{U}\right)$ and downstreamfacing $\left(\varepsilon_{D}\right)$ beams. The colored markers and lines correspond to speed bin-averaged values and lines of best fit on the flood (red) and ebb (blue) tides. The legend indicates best-fit values of the slope, $a$, obtained by regression: i.e., $\varepsilon_{U}=a \varepsilon_{D}$. The solid black line is the 1:1 line, and the dashed lines show the 4:1 and 1:4 relationships.

$\sigma_{e_{i}}$ is assumed to be constant-that is, speed independent. On the other hand, the SF2 method yields an estimate of $\sigma_{e_{i}}$ for each 5- to 7-min data record via $\sigma_{e_{i}}=\sqrt{B_{i} / 2}$. Scatterplots of $\sigma_{N_{i}}$-that is, $\sigma_{e_{i}}$ normalized by the IM estimate for the appropriate beam-are shown in Fig. 10 as a function of both flow speed (left panel) and dissipation rate (right panel). This plot is representative of $\sigma_{N_{i}}$ computed for all the beams at all the ADCP locations, and it not only shows that the speed bin averages are all below the expected value of unity but also that the $\sigma_{N_{i}}$ estimates decrease with increasing flow speed and increasing dissipation rate. The $95 \%$ confidence limits on $\sigma_{N_{i}}$ do increase significantly with flow speed (not shown) and typically include unity. However, the increased uncertainty during strong flows cannot account for the observed systematic bias in the apparent noise levels.

This result is the opposite of the expected trend: flowspeed-dependent decorrelation mechanisms-advection and turbulence-are expected to yield higher Doppler noise levels at higher flow speeds (e.g., Brumley et al. 1991). In addition, the correlations (see supplementary material) were essentially independent of flow speed and near the value of 128 , which is the expected level for high-quality velocity measurements (Gordon 1996). The backscatter amplitude (see supplementary material) did increase slightly with increasing flow speed; however, the signal strength was nearly equal on both phases of the tide. Thus, the variation in the amplitude does not explain the flood-ebb asymmetry in $\sigma_{N_{i}}$ that is apparent in Fig. 10 (and at other sites).

Insight into the low values of $\sigma_{N_{i}}$ was obtained by applying the SF2 method to synthetic time series generated by a linear superposition of sinusoids of frequency $f_{i}$ and amplitude $A_{i}=C_{s} f_{i}^{-5 / 6}$, where $C_{s}$ is a constant dependent on the flow speed and dissipation rate. The phases of the frequency constituents were randomly distributed with uniform probability between 0 and $2 \pi$. The resulting time series yielded Gaussian-distributed velocities and spectral densities proportional to $\varepsilon^{2 / 3} f^{-5 / 3}$, as in real, locally isotropic, homogeneous turbulence (Batchelor 1953, p. 169). Additional noise with a Gaussian distribution and variance $\sigma_{v}^{2}$ was added to the time series to simulate the influence of Doppler noise. To apply the SF2 method, spatial lags $r$ were computed using Taylor's hypothesis, and the $\varepsilon_{i}$ and $\sigma_{e_{i}}$ values were determined via Eq. (9) for $r<6 \mathrm{~m}$. The resulting noise levels $\bar{\sigma}_{N_{i}}$, based on 500 realizations for each specified dissipation rate, are shown in Fig. 11 . The $\bar{\sigma}_{N_{i}}$ values follow the same trend with dissipation rate as the ADCP estimates, but-noticeably-the results from the synthetic time series are also dependent on the maximum frequency $f_{M}$ included in the Fourier series. The dependence of $\bar{\sigma}_{N_{i}}$ on $f_{M}$ suggests that the exclusion of the small-scale motion may be influencing the value of the SF2 $y$ intercept.

Exactly how truncating the Fourier series affects the $D_{L L}$ results is not immediately obvious. Using the same notation as section $5 \mathrm{~b}$, the second-order structure function defined in Eq. (1) can be written as

$D_{L L}=\left\langle u_{0}^{\prime 2}\right\rangle+\left\langle u_{r}^{\prime 2}\right\rangle-2\left\langle u_{0}^{\prime} u_{r}^{\prime}\right\rangle \approx 2\left\langle u_{0}^{\prime 2}\right\rangle-2\left\langle u_{0}^{\prime} u_{r}^{\prime}\right\rangle$

where the assumption of homogeneity has been invoked. The influence of $f_{M}$ on both $\left\langle u_{0}^{\prime 2}\right\rangle$ and $\left\langle u_{0}^{\prime} u_{r}^{\prime}\right\rangle$ was determined using the synthetic time series (as described above) without the addition of Gaussian noise. For a given dissipation rate $\varepsilon_{o}$ and flow speed $|U|$, decreasing $f_{M}$ yielded a smaller variance, whereas the covariance remained constant (see supplementary material). Using values of $\varepsilon_{0}=10^{-4} \mathrm{~W} \mathrm{~kg}^{-1}$ and $U=2 \mathrm{~m} \mathrm{~s}^{-1}$ for the 

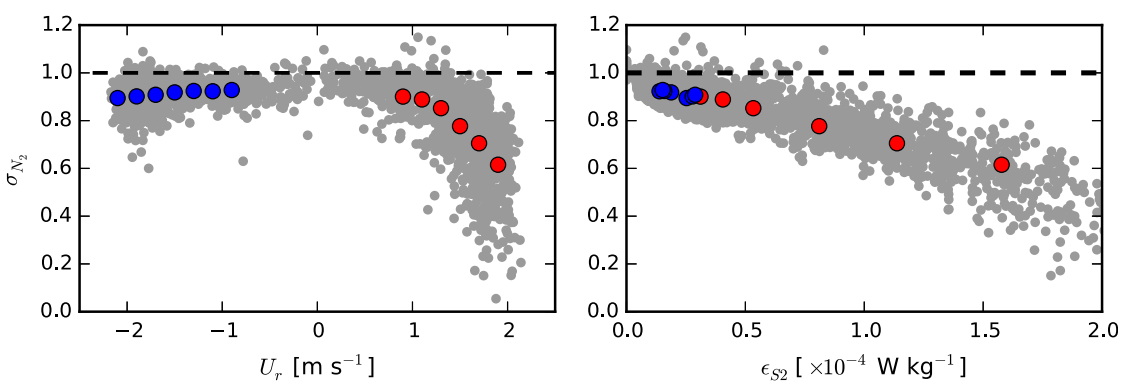

FIG. 10. Normalized Doppler noise standard deviations $\sigma_{N_{i}}$ as a function of (left) flow speed $\left(U_{r}\right)$ and (right) dissipation rate $\left(\varepsilon_{S 2}\right)$ for beam 2 at site $2 \mathrm{a}$. Averages within $0.2 \mathrm{~m} \mathrm{~s}^{-1}$ speed bins are plotted in red and blue for the flood and ebb tides, respectively. The black dashed line represents $\sigma_{N_{i}}=1$, i.e., the expected value based on the velocity spectra at low flow speeds.

synthetic time series, $\left\langle u_{0}^{\prime 2}\right\rangle$ was only about $1 \%$ smaller for $f_{M}=2 \mathrm{~Hz}$ compared to $f_{M}=16 \mathrm{~Hz}$; however, the corresponding decrease in $D_{L L}$ was approximately $10 \%$. Because the variance is independent of $r$, this decrease corresponds to a downward shift of the $D_{L L}$ versus $r^{2 / 3}$ curve, yielding smaller $B_{i}$ values, while the slopes, and hence $\varepsilon_{i}$, remain unaffected. This is consistent with the results plotted in Fig. 12, where the dissipation rates computed from the noise-free synthetic time series are within $2 \%$ of the specified value $\varepsilon_{0}$. The $y$ intercepts, on the other hand, are less than zero and strongly dependent on $f_{M}$ and $\varepsilon_{0}$.

Given the maximum frequency in the time series is $f_{M}$, let $r_{m}=|U| f_{M}^{-1}$ be a representative scale of the unresolved eddies. A modified SF2 method is introduced by assuming that the velocity fluctuations associated with an eddy of size $r_{m}$ scale as

$$
u_{m}^{\prime} \sim\left(\varepsilon r_{m}\right)^{1 / 3},
$$

with corresponding variance $\left\langle u_{m}^{\prime 2}\right\rangle=0.5\left|u_{m}^{\prime}\right|^{2}=0.5 K\left(\varepsilon r_{m}\right)^{2 / 3}$, where $K$ is a scaling factor of $O(1)$. Writing the true variance as the sum of the resolved and unresolved components yields

$$
\widehat{D}_{L L}(r)=C_{2} \varepsilon_{i}^{2 / 3} r^{2 / 3}+2 \tilde{\sigma}_{e_{i}}^{2}-K\left(\varepsilon r_{m}\right)^{2 / 3},
$$

where the tilde is used to distinguish the corrected Doppler noise standard deviation $\tilde{\sigma}_{e_{i}}$ from the value determined via Eq. (9).

The value of $K$ was determined by applying the SF2 method to synthetic time series with various $|U|, \varepsilon_{0}$, and $f_{M}$. Since no noise was added, $\tilde{\sigma}_{e_{i}}^{2}=0$ and it is expected that $B_{i}=-K\left(\varepsilon_{0} r_{m}\right)^{2 / 3}$. The results from all combinations of input parameters are shown in Fig. 13, and it is clear that a linear relationship holds for the range of values considered. By least squares regression, $K=0.764$. Importantly, this value is independent of the mean speed, maximum frequency, and dissipation rate.
Because of the finite ADCP pulse length, eddies smaller than twice the range bin size cannot be resolved. Thus, an ADCP acts as a low-pass filter, having a similar effect to the truncation of a Fourier series. Using $K=0.764$ and $r_{m}=1.06 \mathrm{~m}$ - that is, the minimum resolvable scale along the beam axis with a $0.5-\mathrm{m}$ user-specified vertical cell size and a $20^{\circ}$ beam angle- the corrected Doppler noise levels from the ADCP data are determined via

$$
\tilde{\sigma}_{e_{i}}=\sqrt{\sigma_{e_{i}}^{2}+\frac{K}{2}\left(\varepsilon_{i} r_{m}\right)^{2 / 3}} .
$$

Applying this correction yields $\tilde{\sigma}_{N_{i}}$ values that range between 0.96 and 1.02 for all sites and-importantlyshow no systematic trend with dissipation rate (Fig. 14).

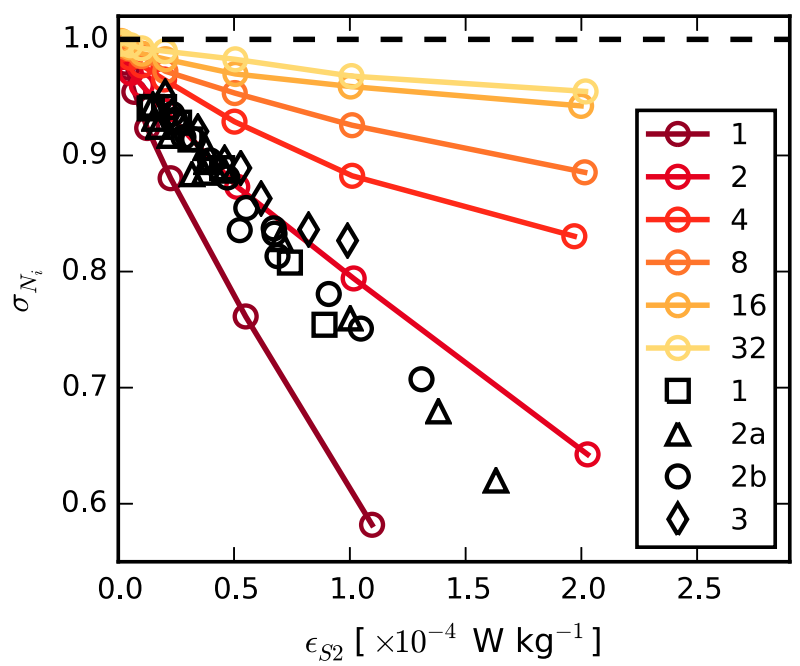

FIG. 11. Normalized noise levels as a function of dissipation rate computed from the SF2 method applied to ADCP data (black markers) and the synthetic time series (colored circles and lines). The ADCP markers represent averages among the four beams and within $0.2 \mathrm{~m} \mathrm{~s}^{-1}$ speed bins. The colored circles represent the average values from 500 realizations of the synthetic time series. The legend indicates the maximum frequency $f_{M}$ (colors, in $\mathrm{Hz}$ ) of the Fourier series and the ADCP deployment sites (black). 

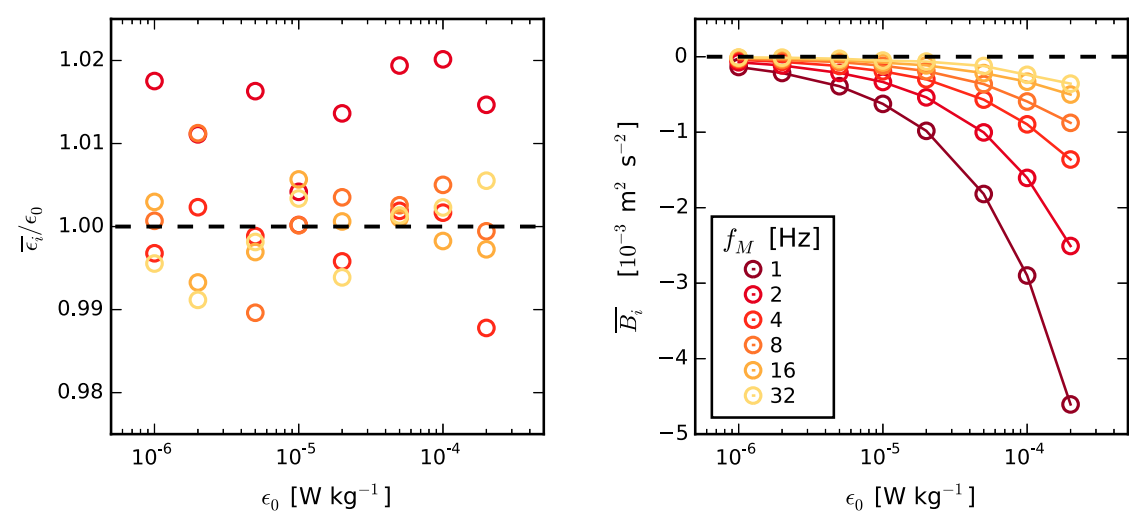

FIG. 12. (left) Average dissipation rate $\left(\bar{\varepsilon}_{i}\right)$ and (right) $y$ intercept $\left(\bar{B}_{i}\right)$ computed by applying the SF2 method to 500 realizations of a synthetic time series generated with a mean flow speed $|U|=2 \mathrm{~m} \mathrm{~s}^{-1}$, dissipation rate $\epsilon_{0}$, and maximum frequency $f_{M}$.

The average and standard deviation of $\tilde{\sigma}_{e_{i}}$ among all beams and speed bins are reported in Table 3. For all sites, the values agree with the estimates determined from the IM.

\section{Conclusions}

Broadband ADCP data from middepth at four locations within a high-flow tidal channel have been used to compare dissipation rates computed using spectral and structure function methods. The results indicate that the spectral integral method (IM) and the second-order structure function (SF2) method yield speed bin-averaged dissipation rates that agree to within $16 \%$, depending on the location and flow direction (ebb vs flood) with the tendency that $\varepsilon_{S 2}<\varepsilon_{\mathrm{IM}}$. The agreement between the methods appears to be independent of the orientation of the ADCP relative to the mean flow direction.

The dissipation rate estimates from the IM and SF2 method provide the following answers to the questions posed in the introduction:

1) Because the $\varepsilon_{I M}$ and $\varepsilon_{S 2}$ estimates agree to within $16 \%$, the factor-of- 2 discrepancy between the ADCP and shear probe measurements observed by McMillan et al. (2016) cannot be attributed to a particular analysis method. Consequently, spatial (i.e., cross channel) variability in turbulence statistics remains the likely explanation for the observed discrepancy.

2) The dissipation rate computed from broadband ADCP measurements is lognormally distributed as expected for high Reynolds number flows, indicating that the intermittency in $\varepsilon$ on 5 -min time scales can be captured using broadband divergingbeam ADCPs.
3) The apparent Doppler noise levels $\sigma_{e_{i}}$ computed using the traditional SF2 method decrease with increasing dissipation rate and are lower than the values determined from the IM. It is shown that the low-pass filtering effect associated with the finite ADCP pulse length (and therefore cell size) results in a $O(1 \%)$ reduction in the measured variance, which in turn shifts the $D_{L L}$ versus $r^{2 / 3}$ curve downward, yielding smaller $\sigma_{e_{i}}$ values while not affecting $\varepsilon_{i}$. A modified SF2 method that accounts for the unresolved variance and involves a constant factor $K$ yields noise levels that are in agreement with the estimates from the IM. The 0.764 value of $K$ used to

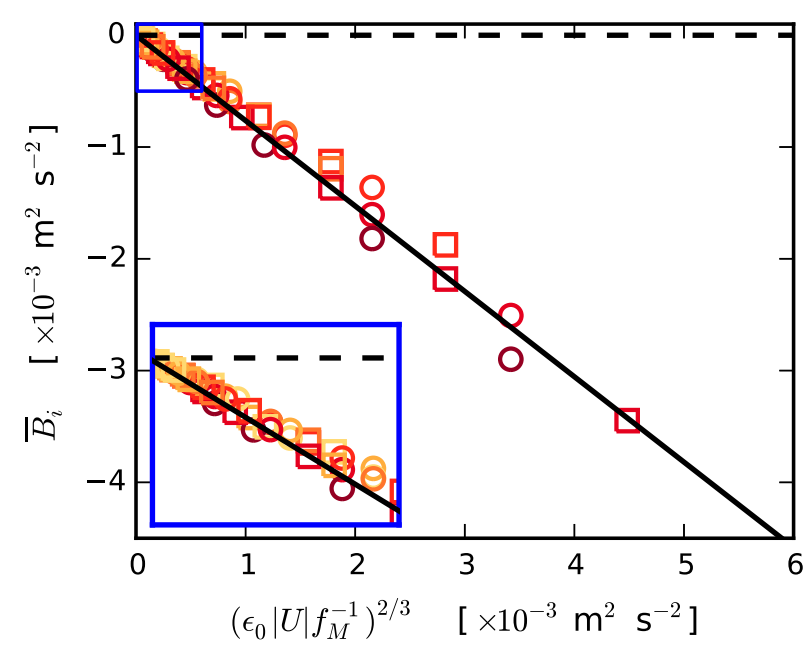

FIG. 13. Average $y$ intercept computed by applying the SF2 method to 500 realizations of a synthetic time series with mean flow speeds of $2 \mathrm{~m} \mathrm{~s}^{-1}$ (circles) and $3 \mathrm{~m} \mathrm{~s}^{-1}$ (squares). The colors correspond to the $f_{M}$ values given in Fig. 12. The solid black line is $\bar{B}_{i}=-K\left(\varepsilon_{0}|U| f_{M}^{-1}\right)^{2 / 3}$ with $K=0.764$ determined using a least squares regression. The inset highlights the data near the origin. The dashed black line is $\bar{B}_{i}=0$. 


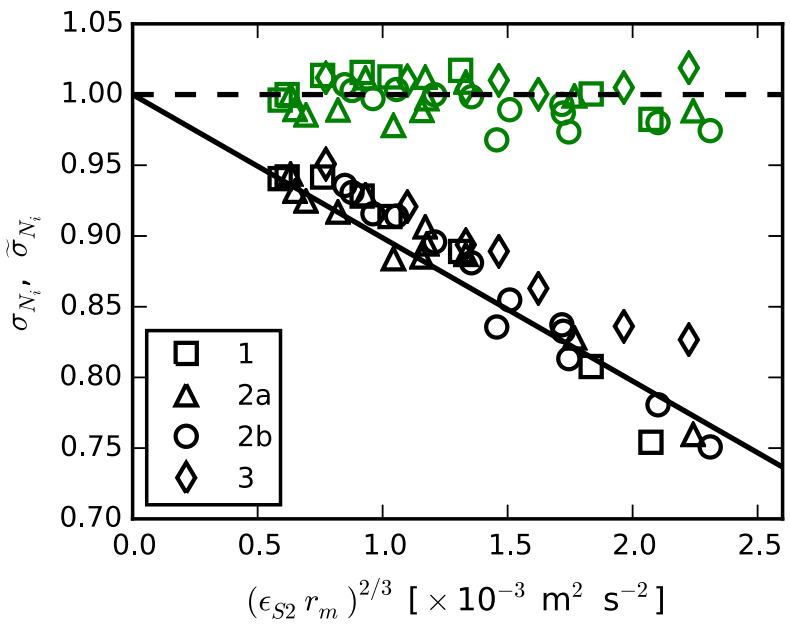

FIG. 14. Normalized Doppler noise standard deviations for all four sites. The plotted values are averages among the four beams, where the black and green markers correspond to the uncorrected $\left(\sigma_{N_{i}}\right)$ and corrected $\left(\tilde{\sigma}_{N_{i}}\right)$ values, respectively. The marker shapes correspond to the sites as indicated in the legend. The solid black line is the expected value of $\sigma_{N_{i}}$ based on the modified SF2 method and the manufacturer-specified accuracy, i.e., $\sigma_{N_{i}}=\left[1-0.5 \sigma_{v}^{-2} K\left(\varepsilon_{S 2} r_{m}\right)^{2 / 3}\right]^{1 / 2}$ with $K=0.764$.

correct the ADCP noise levels was determined from synthetic time series generated by a Fourier series representation of inertial subrange turbulence.

4) The corrected SF2 noise level estimates show no dependence on flow speed or dissipation rate. This result is consistent with the use of a constant spectral noise level in the IM.

The results of this work also support the use of $C_{2}=2.0$ for the SF2 universal constant, which is consistent with the estimate of $\alpha_{1}=0.5$ obtained by Sreenivasan (1995) for the Kolmogorov constant in the streamwise velocity spectrum. Had a value of $C_{2}=2.1$, which has frequently been used in previous studies, been implemented instead, the $\varepsilon_{S 2}$ values would have been $7 \%$ lower and resulted in greater discrepancy with the $\varepsilon_{\mathrm{IM}}$ values. In addition, the third-order SF, which does not involve an empirical constant, showed good agreement with the $\varepsilon_{S 2}$ estimates, with the tendency that $\varepsilon_{S 2}<\varepsilon_{S 3}$. The SF3 method, however, was less robust with a rejection rate that exceeded $72 \%$ at all sites.

The results of this work suggest that the SF2 method can be more robust in comparison to the IM when estimating $\varepsilon$ on 5 -min time scales from broadband ADCP data in high-speed tidal flows. For these data, the SF2 method yields reliable estimates of $\varepsilon$ for up to $90 \%$ of the ensembles, compared with a maximum of $60 \%$ for the IM.

Regardless of the choice of method, broadband ADCPs can be used to estimate $\varepsilon$ remotely, provided stratification and vertical velocity shear are both sufficiently weak. It is expected that these results, which specifically considered the estimation of $\varepsilon$ on shorter time scales corresponding to intermittency (in the statistical sense), can be extended to examine variability on longer time scales corresponding to tidal frequencies. Thus, these conclusions are particularly relevant for tidal energy developments, for which estimates of the turbulence levels at multiple scales are important for resource assessment and engineering design.

Acknowledgments. We thank Richard Cheel, Tristan Guest, and Greg Trowse for assistance in the field. The motivation for the paper was provided by Rolf Lueck, and valuable input on the interpretation of the results was given by Len Zedel. We also acknowledge the captains and crew of the vessels-Saint Nicholas II, Island Lady $G$, Nova Endeavour, and Great Expectations XLused during the completion of this work. Funding was provided by the Offshore Energy Research Association, the Natural Sciences and Engineering Research Council of Canada, Natural Resources Canada, and Killiam Trusts.

\section{REFERENCES}

Batchelor, G. K., 1953: The Theory of Homogeneous Turbulence. Cambridge University Press, 197 pp.

Brumley, B. H., R. G. Cabrera, K. L. Deines, and E. A. Terray, 1991: Performance of a broad-band acoustic Doppler current profiler. IEEE J. Oceanic Eng., 16, 402-407, doi:10.1109/ 48.90905.

Collignon, A. G., and M. T. Stacey, 2013: Turbulence Dynamics at the Shoal-Channel Interface in a Partially Stratified Estuary, J. Phys. Oceanogr., 43, 970-989, doi:10.1175/ JPO-D-12-0115.1.

de Kármán, T., and L. Howarth, 1938: On the statistical theory of isotropic turbulence. Proc. Roy. Soc. London, 164A, 192-215, doi:10.1098/rspa.1938.0013.

Gordon, R. L., 1996: Acoustic Doppler current profiler: Principles of operation; A practical primer. 2nd ed. RD Instruments Tech. Rep. P/N 951-6069-00, 52 pp.

Grant, H. L., R. W. Stewart, and A. Moilliet, 1962: Turbulence spectra from a tidal channel. J. Fluid Mech., 12, 241-268, doi:10.1017/S002211206200018X.

Gunawan, B., V. S. Neary, and J. Colby, 2014: Tidal energy site resource assessment in the East River tidal strait, near Roosevelt Island, New York, New York. Renewable Energy, 71, 509-517, doi:10.1016/j.renene.2014.06.002.

Hay, A. E., J. M. McMillan, R. Cheel, and D. J. Schillinger, 2013: Turbulence and drag in a high Reynolds number tidal passage targeted for in-stream tidal power. Oceans 2013, IEEE, 361-370.

Kolmogorov, A. N., 1941a: Dissipation of energy in the locally isotropic turbulence. Dokl. Akad. Nauk SSSR, 32, 19-21. , 1941b: The local structure of turbulence in incompressible viscous fluid for very large Reynolds numbers. Dokl. Akad. Nauk SSSR, 30, 301-305.

Lanckriet, T., and J. A. Puleo, 2013: Near-bed turbulence dissipation measurements in the inner surf and swash zone. J. Geophys. Res. Oceans, 118, 6634-6647, doi:10.1002/2013JC009251. 
Lorke, A., 2007: Boundary mixing in the thermocline of a large lake. J. Geophys. Res., 112, C09019, doi:10.1029/2006JC004008.

— bulence measurements in the bottom boundary layer. J. Atmos. Oceanic Technol., 22, 1821-1828, doi:10.1175/JTECH1813.1.

Lucas, N. S., J. H. Simpson, T. P. Rippeth, and C. P. Old, 2014: Measuring turbulent dissipation using a tethered ADCP. J. Atmos. Oceanic Technol., 31, 1826-1837, doi:10.1175/JTECH-D-13-00198.1.

Malinka, C. E., 2013: Acoustic detection ranges and baseline ambient noise measurements for a marine mammal monitoring system at a proposed in-stream tidal turbine site: Grand Passage, Nova Scotia. B.Sc. thesis, Dept. of Biology, Dalhousie University, $73 \mathrm{pp}$.

McMillan, J. M., A. E. Hay, R. G. Lueck, and F. Wolk, 2016: Rates of dissipation of turbulent kinetic energy in a high Reynolds number tidal channel. J. Atmos. Oceanic Technol., 33, 817837, doi:10.1175/JTECH-D-15-0167.1.

Milne, I. A., R. N. Sharma, R. G. J. Flay, and S. Bickerton, 2013: Characteristics of the turbulence in the flow at a tidal stream power site. Philos. Trans. Roy. Soc. London, 371A, 20120196, doi:10.1098/rsta.2012.0196.

Mohrholz, V., H. Prandke, and H. U. Lass, 2008: Estimation of TKE dissipation rates in dense bottom plumes using a Pulse Coherent Acoustic Doppler Profiler (PC-ADP)-Structure function approach. J. Mar. Syst., 70, 217-239, doi:10.1016/ j.jmarsys.2007.03.004.

Monin, A. S., and A. M. Yaglom, 1975: Statistical Fluid Mechanics: Mechanics of Turbulence. Vol. 2, MIT Press, $869 \mathrm{pp}$.

Nuttall, A. H., 1971: Spectral estimation by means of overlapped Fast Fourier Transform processing of windowed data. NUSC Rep. 4169, 40 pp. [Available online at http://www.dtic.mil/gettr-doc/pdf?AD = AD0739315.]

Obukhov, A., 1941: On the energy distribution in the spectrum of a turbulent flow. Dokl. Akad. Nauk SSSR, 32, 19-21.

Osalusi, E., J. Side, and R. Harris, 2009: Reynolds stress and turbulence estimates in bottom boundary layer of Fall of Warness. Int. Commun. Heat Mass Transfer, 36, 412-421, doi:10.1016/j.icheatmasstransfer.2009.02.004.

Pond, S., R. W. Stewart, and R. W. Burling, 1963: Turbulence spectra in the wind over waves. J. Atmos. Sci., 20, 319-324, doi:10.1175/1520-0469(1963)020<0319:TSITWO>2.0.CO;2.

Pope, S. B., 2000: Turbulent Flows. Cambridge University Press, $771 \mathrm{pp}$.
Richard, J.-B., J. Thomson, B. Polagye, and J. Bard, 2013: Method for identification of Doppler noise levels in turbulent flow measurements dedicated to tidal energy. Int. J. Mar. Energy, 3-4, 52-64, doi:10.1016/j.ijome.2013.11.005.

Saddoughi, S. G., and V. Veeravalli, 1994: Local isotropy in turbulent boundary layers at high Reynolds number. J. Fluid Mech., 268, 333-372, doi:10.1017/S0022112094001370.

Sauvageot, H., 1992: Radar Meteorology. Artech House, 366 pp.

Simpson, J. H., P. J. Wiles, and B. J. Lincoln, 2011: Internal seiche modes and bottom boundary-layer dissipation in a temperate lake from acoustic measurements. Limnol. Oceanogr., 56, 1893-1906, doi:10.4319/lo.2011.56.5.1893.

—, N. S. Lucas, B. Powell, and S. C. Maberly, 2015: Dissipation and mixing during the onset of stratification in a temperate lake, Windermere. Limnol. Oceanogr., 60, 29-41, doi:10.1002/ lno.10008.

Sreenivasan, K. R., 1995: On the universality of the Kolmogorov constant. Phys. Fluids, 7, 2778-2784, doi:10.1063/1.868656.

Stacey, M. T., S. G. Monismith, and J. R. Burau, 1999: Measurements of Reynolds stress profiles in unstratified tidal flow. J. Geophys. Res., 104, $10933-10949$, doi:10.1029/ 1998JC900095.

Taylor, G. I., 1935: Statistical theory of turbulence. Proc. Roy. Soc. London, 151A, 421-444, doi:10.1098/rspa.1935.0158.

Thomson, J., 2012: Wave breaking dissipation observed with "SWIFT" drifters. J. Atmos. Oceanic Technol., 29, 1866-1882, doi:10.1175/JTECH-D-12-00018.1.

_ - B. Polagye, V. Durgesh, and M. C. Richmond, 2012: Measurements of turbulence at two tidal energy sites in Puget Sound, WA. IEEE J. Oceanic Eng., 37, 363-374, doi:10.1109/ JOE.2012.2191656.

Whipple, A. C., and R. A. Luettich, 2009: A comparison of acoustic turbulence profiling techniques in the presence of waves. Ocean Dyn., 59, 719-729, doi:10.1007/s10236-009-0208-3.

Wiles, P. J., J. H. Simpson, and T. P. Rippeth, 2005: A novel technique for measuring mixing using acoustic Doppler current profilers (ADCPs). Proceedings of the IEEE/OES Eighth Conference on Current Measurement Technology, IEEE, 9-13, doi:10.1109/CCM.2005.1506324.

— novel technique for measuring the rate of turbulent dissipation in the marine environment. Geophys. Res. Lett., 33, L21608, doi:10.1029/2006GL027050. 